\title{
Diagnóstico desde las competencias comunicativas sobre el aprendizaje del idioma inglés de estudiantes del Instituto Técnico Industrial Centro Don Bosco
}

\section{Diagnosis from the Communicative Competences on the Learning of the English language of the Students of the Don Bosco Center Industrial Technical Institute}

Jaime Sánchez Medina*

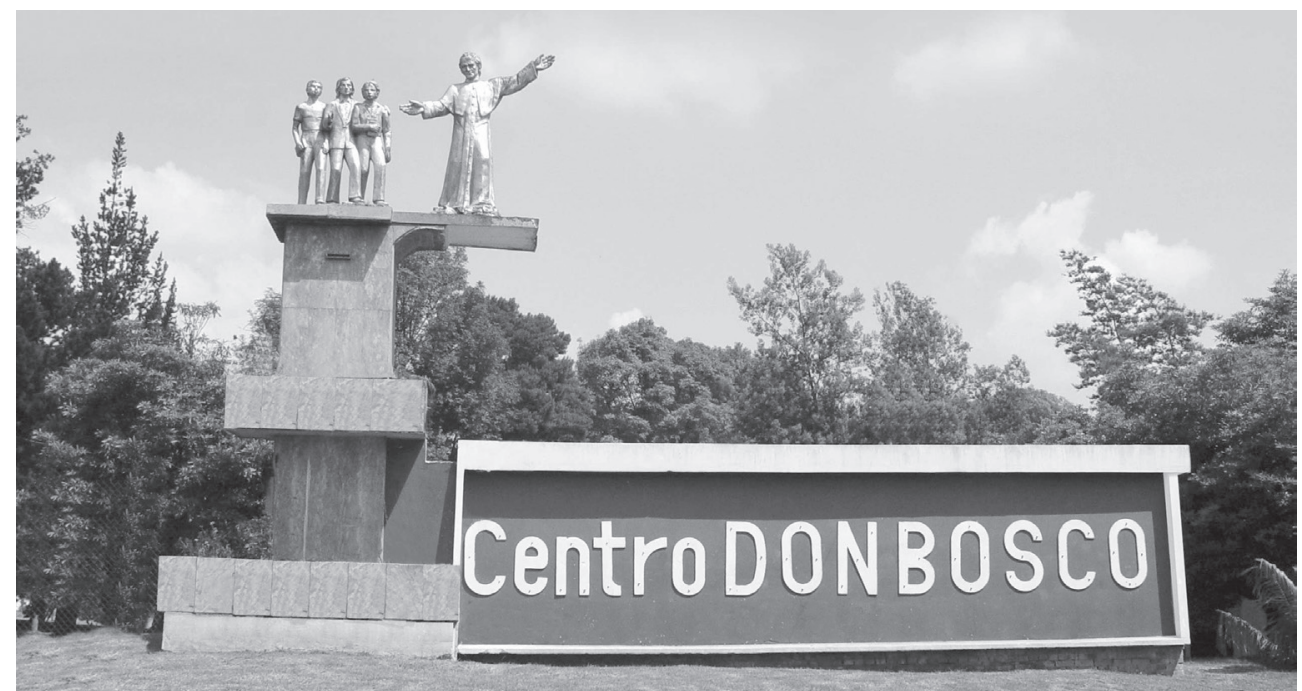

\section{Resumen}

El estudio se refiere al aprendizaje del idioma inglés con fines específicos, proceso en el que se fortalece el desarrollo de una competencia comunicativa específica propia de un saber hacer (know-how) en un área específica del conocimiento. Su objetivo fue determinar, mediante observación participante y un cuestionario autoaplicado, las competencias comunicativas específicas para el aprendizaje de inglés que necesitan

Citar este artículo como: Sánchez Medina, J. (2017). Diagnóstico desde las competencias comunicativas sobre el aprendizaje del idioma inglés de estudiantes del Instituto Técnico Industrial Centro Don Bosco. Revista Papeles, 9(17), 87-95.

Fecha de recibido: febrero 5 de 2017.

Fecha de aceptación: mayo 10 de 2017.

* Especialista en Docencia Universitaria de la Universidad Militar Nueva Granada (UMNG). Licenciado en Idiomas (español e inglés) de la Universidad Antonio Nariño. Amante y defensor del buen uso de la palabra en la oralidad como en la escritura. Ha sido docente en instituciones educativas de la Sociedad Salesiana en Bogotá, D. C. Correo electrónico: jaimeor.sanchezm@gmail.com 
desarrollar los estudiantes de los programas de formación técnica para el trabajo y el desarrollo humano del Instituto Técnico Industrial Centro Don Bosco. Su enfoque fue mixto. Tanto sus resultados como su discusión convocan propuestas que se centren en establecer estrategias didácticas específicas para fortalecer el gusto y las competencias de lectura, escritura y expresión oral en inglés con fines específicos.

Palabras clave: aprendizaje, inglés, educación, desarrollo humano, competencias comunicativas, estrategias didácticas, interpretación, documentos técnicos.

\begin{abstract}
The study refers to the learning of the English language for specific purposes, a process in which the development of a specific communicative competence proper to the know-how of a specific area of knowledge, is strengthened. Its objective was to determine, through participant observation and a self-applied questionnaire, the specific communicative competences for the learning of English that the students of the technical training programs for work and human development of the Don Bosco Center Industrial Technical Institute need to develop. His approach was mixed. Both its results and its discussion call for proposals that focus on establishing specific teaching strategies to strengthen the taste and skills of reading, writing and speaking in English for specific purposes.
\end{abstract}

Key words: learning English, education for work and human development, communicative competences, didactic strategies, interpretation of technical documents.

\title{
Introducción
}

En el aprendizaje de inglés se fortalece el desarrollo de las competencias comunicativas: lingüística, pragmática, funcional y sociolingüística mediante estrategias didácticas específicas que favorecen la relación dialógica estudiante-conocimiento-docente con el objetivo de dar respuesta a las necesidades e intereses personales y socioculturales. El Ministerio de Educación Nacional (MEN, 2006a; MEN, 2013) define competencia como "El conjunto de saberes, conocimientos, destrezas y características individuales que permite a una persona realizar acciones en un contexto determinado". Las necesidades e intereses de aprendizaje permiten entender el inglés como lengua extranjera (EFL — por sus siglas en inglés-), que busca el desarrollo integral de las habilidades comunicativas (Moreno, 1994) con el fin de lograr una comunicación intercultural. Patricio y María (2014) hablan de enseñanza de inglés para el desarrollo de competencias comunicativas interculturales; dado que la cultura tiene un papel relevante en el proceso de enseñanza-aprendizaje de una lengua. También se entiende el inglés con propósitos específicos (ESP, por sus siglas en inglés). Fiorito (2005) expresa que "ESP students are usually adults who already have some acquaintance with English and are learning the language in order to communicate a set of professional skills and to perform particular job-related functions". Freire (2010) invita a la reflexión cuando señala que

El sentido -o uno de los sentidos principales- que me motiva a tratar este tema [contexto concreto-contexto-teórico] es el de subrayar la importancia de la relación en todo lo que hacemos en nuestra experiencia existencial en cuanto experiencia social e histórica. La importancia 
de la relación entre las cosas, de los objetos entre sí, de las palabras entre ellas en la composición de las frases y de éstas entre sí en la estructura del texto (...) Lo importante que resultan, en suma, las relaciones entre educandos y educadoras, sujetos cognoscentes y objetos cognoscibles. (p. 123)

El Ministerio de Educación Nacional expresa que las competencias laborales específicas están "orientadas a habilitar a un individuo para desarrollar funciones productivas propias de una ocupación o funciones comunes a un conjunto de ocupaciones" (MEN, 2003). $\mathrm{Al}$ respecto, el Estado mediante la Ley 1064 de 2006 reconoce la Educación para el Trabajo y el Desarrollo Humano (MEN, 2006b; MEN, 2009) como una apuesta para el fortalecimiento de las políticas públicas educativas que respondan a las necesidades de la apertura comercial con la globalización y la continua industrialización de los mercados (Fedesarrollo, 2004). La enseñanza del idioma inglés está articulada a partir de los lineamientos del Marco Común Europeo (Centro Virtual Cervantes, 2002-2007) por el Ministerio de Educación Nacional mediante el Decreto No. 3870-2006 (MEN, 2006c), la Secretaría de Educación Distrital y el SENA. Además, para el fortalecimiento a nivel nacional del desarrollo de las competencias en inglés, el MEN (MEN, 2013) estableció el Plan Nacional de Inglés 2015-2025 y, a través del Ministerio de Educación Nacional, en su documento Series Guía 22 estableció los "Estándares Básicos de Competencias en Lenguas Extranjeras: Inglés" (MEN, 2006a), y específicamente para las estrategias de aprendizaje de inglés estableció el desarrollo de las competencias comunicativas a la luz del plan curricular y el proyecto educativo institucional (PEI). El Monterrey I.T. (s.f) señala que la estrategia didáctica es el conjunto de procedimientos apoyados en técnicas de enseñanza, que tienen por objeto llevar a buen término la acción didáctica, es decir, alcanzar los objetivos del aprendizaje (p. 5). En las estrategias didácticas para la enseñanza, el discurso pedagógico está en relación de liderazgo docente-estudiante, y para el aprendizaje en relación estudiantedocente, en donde el estudiante se apropia del proceso de autoformación con el acompañamiento del docente - atendiendo al modelo autoestructurante propuesto por Not (1983 y 1992) y citado por De Zubiría (1994) en su texto ¿Qué modelo pedagógico subyace a su práctica educativa?- - En cualquiera de las estrategias, el docente es quien debe atender las diferencias individuales de aprendizaje de cada estudiante (Gardner, 1995) y los factores situacionales y sociales. A este respecto, Rubbin (1987, citado en Ellis, 1996) establece que "Learning strategies are strategies which contribute to the development of the language system which the learner constructs and affect learning directly" (p. 528), en que, para garantizar al máximo la calidad educativa, se debe contextualizar la acción pedagógica, tanto a nivel grupal como individual del estudiante. Ellis (1996) expresa que estrategias de aprendizaje son estrategias que contribuyen al desarrollo del sistema lingüístico que el estudiante construye, incidiendo en el aprendizaje directamente $y$, luego expone que

(...) individual learner differences (beliefs, affective states, general factors, and previous learning experiences) together with various situational factors (the target language being studied, whether the setting is formal or informal, the nature of the instruction, and the specific tasks learners are asked to perform) determine the learner's choice of learning strategies. (p. 471)

El Instituto Técnico Industrial Centro Don Bosco es una institución educativa localizada en la ciudad capital Bogotá D. C., República de Colombia, cuya misión contempla el acompañamiento a los jóvenes "en el desarrollo del pensamiento científico y técnico y tecnológico que posibilite un trabajo competente y digno" 
(Instituto Técnico Industrial Centro Don Bosco, 2016) a través de la acción pedagógica, epistemológica y antropológica que fortalezca el desarrollo de las competencias comunicativas (MEN, 2006a) para el desempeño en la economía y el talento humano (Agüero, 2009, p. 5) y su aprendizaje como una oportunidad para el desarrollo personal, laboral y social. En este escenario surge el interrogante en el que se enmarca este estudio, como es: ¿qué competencias comunicativas específicas necesitan desarrollar para el aprendizaje del inglés los estudiantes de los programas de educación técnica para el trabajo y el desarrollo humano del Instituto Técnico Industrial Centro Don Bosco?

\section{Objetivos}

El objetivo general fue determinar las competencias comunicativas específicas para el aprendizaje de inglés que necesitan desarrollar los estudiantes de los programas de educación técnica y desarrollo humano del Instituto Técnico Industrial Centro Don Bosco. Los objetivos específicos fueron: primero, realizar una descripción sociodemográfica de la población objeto del estudio; luego, aplicar a ella un cuestionario autoaplicado que permitiera corroborar o desvirtuar las creencias acerca del aprendizaje del inglés (Martínez, 2005) identificadas mediante la observación en los ambientes pedagógicos; y finalmente, complementar resultados de ambas etapas para dejar planteada un propuesta de optimización de las didácticas del proceso enseñanza-aprendizaje de esta lengua extranjera.

\section{Metodología}

El estudio siguió un enfoque mixto (Hernández, Fernández y Baptista, 2006) dado sus dos componentes: cualitativo y cuantitativo. Una primera etapa de observación participante (Universidad de los Andes, s.f.) en un

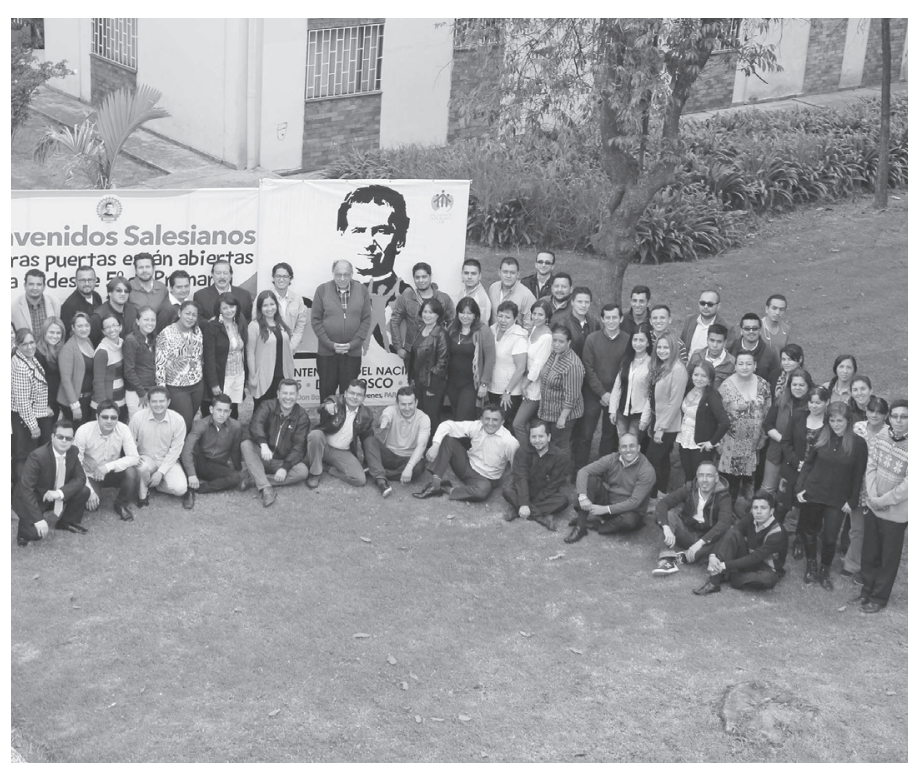

periodo de cuatro meses, una segunda etapa de aplicación de un cuestionario autoaplicado (Núñez, 2007) a 151 estudiantes, con consentimiento informado de participación libre y autónoma, y un tercer momento en que se elaboró una descripción de la primera etapa y un análisis del resultado del cuestionario para complementar resultados. Hernández, et al. (2006) destacan que "el enfoque mixto va más allá de la simple recolección de datos de diferentes modos sobre el mismo fenómeno, implica desde el planteamiento del problema mezclar la lógica inductiva y la deductiva"; y Teddlie y Tashakkori (citado en Hernández, et al., 2006) afirman que "un estudio mixto lo es en el planteamiento del problema, la recolección y análisis de datos, y en el reporte del estudio"; y Creswell (citado en Hernández, et al., 2006) expresa que "los [problemas] que requieren ser explorados para obtener un entendimiento profundo, 'empatan' más con un diseño cualitativo". 


\section{Análisis de resultados}

La etapa de observación participante de este estudio permitió identificar la existencia de una creencia (Martínez, 2005) de desinterés, apatía o sentimiento de suficiencia frente al aprendizaje de inglés por parte de los estudiantes; e incluso por parte de algunos docentes. La baja participación en los ejercicios al interior del aula o trabajo autónomo relacionados especialmente con actividades para la lectura, la escritura y la expresión oral obedecen a la debilidad en el conocimiento de inglés básico, y el cansancio laboral con el que los estudiantes llegan a clases hace pensar en la necesidad de usar didácticas más dinámicas.

A continuación son presentados los resultados más relevantes porcentualmente arrojados por la tabulación del cuestionario, con el fin de ir construyendo los resultados mismos desde la visión del enfoque mixto. La información sociodemográfica arrojada por la tabulación del cuestionario aplicado corresponde a 151 personas que respondieron el cuestionario: el $96,7 \%$ fueron hombres, el 1,3\% mujeres y el $2,0 \%$ no respondieron este ítem. Con respecto a la edad, el mayor porcentaje $(42,4 \%)$ correspondió a la comprendida entre 20-30 años, seguida por el $23,2 \%$ de los 31-40 años y el menor porcentaje (2,6\%) fue de los 41-51 años. El 7,3\% no respondió este ítem. En la distribución de carreras técnicas, el 26,5\% pertenece a la carrera de redes eléctricas residenciales e industriales, el 23,8\% a mecánica industrial, ajuste y soldadura, y el valor más bajo fue para electrónica de potencia, con $11,9 \%$. Respecto del grado de escolaridad, el 2,0\% había cursado hasta noveno grado, el 55,6\% básica secundaria y media completa, y el $29,8 \%$ alguna carrera técnica ya certificada.

Ante la pregunta " $¿$ Usted considera importante aprender el idioma inglés dentro de su carrera técnica?", el 96,7\% respondió "Sí", el 0,7\% "No", y el 2,6\% no respondió a este ítem. Frente a la proposición "Considero que mi desempeño actual en el idioma inglés es:" se halló que los porcentajes más altos fueron: $43,7 \%$ en nivel bajo para hablar, $40,4 \%$ en nivel medio para escuchar, $44,4 \%$ en nivel medio para escribir y $45,0 \%$ en nivel medio para leer. El ítem núm. 6 correspondió a la proposición "Dentro de los estudios de una carrera técnica, considero que aprender inglés es importante para:", en donde se obtuvo que el $48,3 \%$ lo consideraba importante para escribir y el $23,8 \%$ para leer; 16,6\% no respondió este ítem. En el ítem núm. 7 se propuso "Dentro de la materia de inglés, en su carrera técnica, en orden de menor a mayor, en una escala de 1 a 3 , en donde $1=$ Nada importante, $2=$ importante; y $3=$ Muy importante; usted considera que se debería desarrollar más la habilidad comunicativa para:". El descriptor de "muy importante" fue otorgado para hablar $(61,6 \%)$, leer $(58,9 \%)$, escribir $(49,7 \%)$ y escuchar $(49,0 \%)$.

Frente a la proposición "Enumere 1 como el más importante en la siguiente lista, en relación con la expresión "Aprender el idioma inglés dentro de mi carrera técnica me ha de servir para:"', el resultado en orden descendente fue el 32,5\% para "permitirme hacer", el 13,9\% para "resolver" y el 12,6\% para "descubrir". Ante la proposición, en el ítem 9: "Considero que una actividad que me permitiría aprender más y de una manera más fácil el idioma inglés es:", el resultado en orden descendente fue "Mayor práctica audiovisual" (18,5\%), "Mayor práctica de Role Play" (16,6\%) y "Mayor práctica oral" $(11,9)$. "En mi vida me ha gustado o me gustaría tomar un curso de inglés" fue la proposición del ítem 10, en donde el 96,0\% respondió "Sí", el 3,3\% respondió "No" y el 0,7\% no respondió a este ítem. Finalmente, en el ítem 11\%, frente a la proposición "Considero que para vivir un buen proceso de aprendizaje de inglés básico en mi carrera técnica, el tiempo de clase por semana debería ser", se halló que los estudiantes reclaman más de 90 minutos de clase por semana $(45,0 \%)$. 
La etapa de observación participante de este estudio permitió identificar la existencia de una creencia (Martínez, 2005) de desinterés, apatía o sentimiento de suficiencia frente al aprendizaje de inglés por parte de los estudiantes; e incluso por parte de algunos docentes.
Una vez comparados los resultados del ejercicio de observación participante con los arrojados por los de la tabulación del cuestionario, se identificaron: interés y buena disposición para aprender inglés. $\mathrm{Y}$ algunas necesidades prioritarias: necesidad de desarrollar competencias comunicativas para la lectura y la escritura para favorecer el ejercicio de interpretación de documentos técnicos y el procesamiento de información técnica, y para la expresión oral del inglés en contextos laborales; necesidad de fortalecer el gusto por el aprendizaje de inglés mediante didácticas dinámicas que fortalezcan el aprendizaje significativo.

\section{Resultados}

La etapa de observación participante se inició en el mes de agosto de 2016 y culminó en el mes de noviembre del mismo año. Del universo de estudio de ciento cincuenta y un (151) estudiantes, tan solo dos (2) correspondían al género femenino. La totalidad del universo cumplía el rol de trabajadores-estudiantes, quienes en su mayoría ejercían labores de carácter práctico-técnico; razón por la cual algunos de ellos al momento de iniciar las clases presentan notables índices de cansancio

Tabla 1. Resultados cualitativos

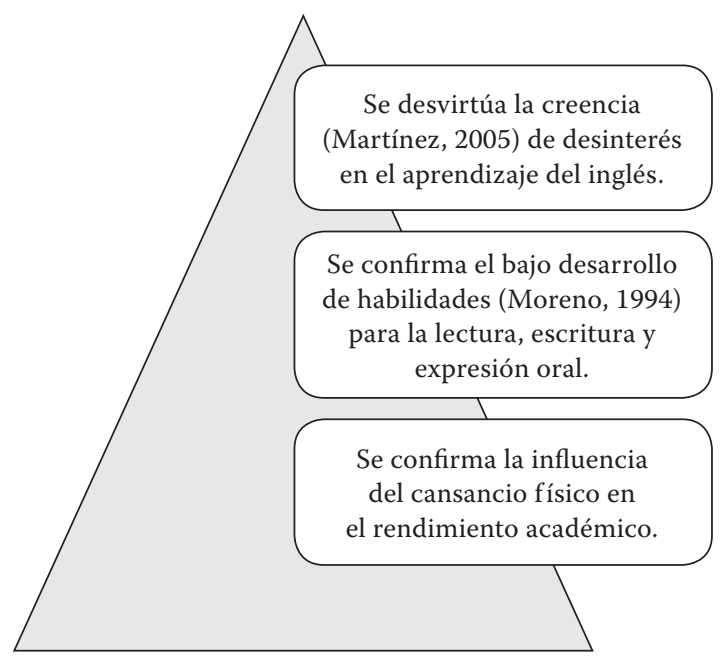

Fuente: Elaboración Propia físico, que van desde dolores físicos hasta la somnolencia. Se enfatizó la observación en los procesos de cumplimientos de tareas o ejercicios al interior del aula y en los talleres de práctica formativa, control de trabajo autónomo y participación oral en clase; desde donde se identificó debilidad en el inglés básico, tanto para hablar como para escribir y leer. Los resultados del estudio desde el enfoque mixto (Hernández, Fernández y Baptista, 2006) son:

Tabla 2. Resultados cuantitativos

Dentro de su carrera técnica, en el aprendizaje de inglés se debería desarrollar más la habilidad comunicativa para:

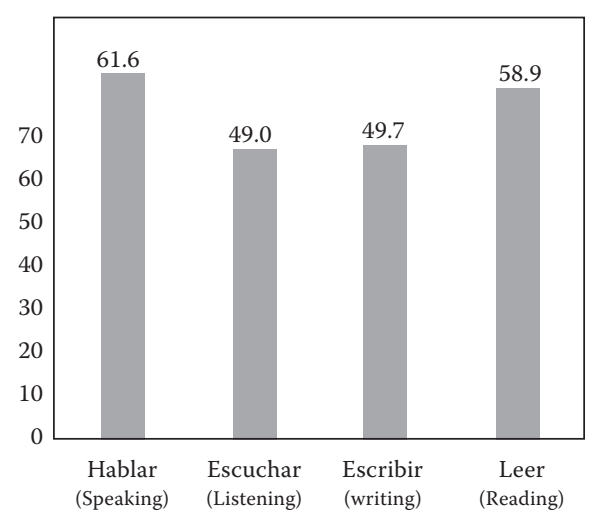

Fuente: Elaboración Propia 


\section{Discusión}

Este estudio permitió identificar que síhayinterés y buena disposición para aprender inglés por parte de los estudiantes de los programas técnicos laborales del Centro Don Bosco. También se reconocieron las necesidades de desarrollar la competencia comunicativa para la lectura y la escritura con el fin de favorecer el ejercicio de interpretación de documentos técnicos y el procesamiento de información técnica, así como la expresión oral del inglés en contextos laborales. Además se debe fortalecer el gusto por el aprendizaje del inglés mediante didácticas dinámicas que fortalezcan el aprendizaje significativo. Teniendo en cuenta que el proceso de enseñanzaaprendizaje del inglés técnico (Fiorito, 2005) en el Centro Don Bosco en forma presencial fue instaurado en febrero de 2016, estos resultados se consolidan como una oportunidad para dinamizar las estrategias didácticas en la especificidad de cada una de las necesidades e intereses dentro de todos y cada uno de los programas técnico laborales. Por supuesto, el interés del ejercicio docencia-investigación debe continuar con el objetivo de consolidar cada día más las estrategias tanto pedagógicas como didácticas. La discusión queda abierta para profundizar a través de nuevos estudios y reflexiones alrededor de las siguientes preguntas: ¿cómo optimizar el proceso de aprendizaje del inglés?, y ¿qué didácticas específicas se deben desarrollar para la interpretación de documentos técnicos, el procesamiento de información técnica y para la expresión oral del inglés en contextos laborales en los programas técnicos laborales? (Bonamy, 2012). Cualquier acercamiento a develar alguno o todos los interrogantes dejará abierto el interés por estructurar en profundidad un proyecto de investigación-intervención.

\section{Conclusiones}

Los resultados del presente estudio-diagnóstico motivan la necesidad de establecer estrategias didácticas específicas que desarrollen las competencias comunicativas de lectura y escritura para la interpretación de documentos técnicos, el procesamiento de información técnica y la competencia comunicativa para la expresión oral del inglés en contextos laborales (Fiorito, 2005), así como para el reconocimiento de la importancia y el gusto o gozo (Wagensberg, 2007) por aprender inglés, desde la pedagogía constructivista (Hernández, 2008) y la teoría del aprendizaje significativo (Ausubel, 1983).
Estrategias didácticas que potencien la comprensión de textos, la traducción científica, los elementos de cohesión al interior de la estructura del texto y el proceso de lectura crítica (Batista, et al, 2007, pp. 146-173), tales como el aprendizaje basado en problemas (ABP [Restrepo, 2005]), el seminario alemán (Pérez, 2010), los mapas mentales (Buzan, s.f.), o el uso del video como medio de aprendizaje (Fernández y Bravo, s.f.). La flexibilidad evaluativa debe de integrar la heteroevaluación, la coevaluación y la autoevaluación (Espacio Europeo de Educación Superior, 2009).

\section{Referencias}

Agüero, C. (2009). Educación, Cultura y Desarrollo en la Globalización, Universalidad cultural de la educación para el desarrollo. Sapiens, Revista Universitaria de Investigación, 1(10), 5.
Ausubel, D. (1983). Teoría del aprendizaje significativo [en línea], disponible en: http:// delegacion233.bligoo.com.mx/media/ users/20/1002571/files/240726/Aprendizaje_ significativo.pdf, recuperado: marzo, 2017 
Batista, J.; Alburguez, M. y T. León, M. (2007). Estudio Diagnóstico Sobre la Enseñanza del Inglés Técnico en Ingeniería. Laurus, 13(25), 146-173.

Bonamy, D. (2012), English for communication in the workplace, [en línea], disponible en: http: //www.pearsonlongman. $\mathrm{com} /$ vocationalenglish/pdf/articles/English_ for_communication_in_the_workplace.pdf, recuperado: marzo, 2017.

Buzan, T. (s.f). El libro de los mapas mentales, resumen por Mercedes González [en línea], disponible en http://docencia.fca.unam.mx / esosa/archivos/mapas_mentales.pdf, recuperado: marzo, 2017.

Centro Virtual Cervantes (2002-2007), Marco Común Europeo de Referencia para las Lenguas, [en línea], disponible en cvc.cervantes. es/ensenanza/biblioteca_ele/marco/, recuperado: marzo, 2017.

De Zubiría, J. (1994). ¿Qué modelo pedagógico subyace en su práctica pedagógica? [en línea], disponible en: http://www.uovirtual.com.mx/ moodle/lecturas/tendepeco/1/1.pdf, recuperado: marzo, 2017.

Ellis, R. (1996) The Study of Second Language Acquisition [El Estudio de la Adquisición de una Segunda Lengua], Oxford University Press, Hong Kong.

Espacio Europeo de Educación Superior (2009). La evaluación como aprendizaje, ediciones Akal, Madrid.

Fedesarrollo (2004). Tratado de libre comercio Colombia-Estados Unidos: Análisis del impacto económicosobrelaregiónBogotá-Cundinamarca [en línea], disponible en: http://www.fedesarrollo.org.co/wp-content/uploads/2011/08/ Impacto-econ \% C $3 \%$ B 3 mico-del-TLCcon-Estados-Unidos-en-la-regi\%C3\%B3nBogot\%C3\%A1-Cundinamarca-informe-final. pdf

Fernández, RL., Bravo J. (s.f.). El video en la enseñanza del inglés técnico [En línea], disponible en: http://www.ice.upm.es/wps/ jlbr/Documentacion/Vid_Ingles_Tec.pdf

Fiorito, L. (2005). Teaching English for Specific Purposes (ESP) [en línea], disponible en: http:// www.usingenglish.com/articles/teachingenglish-for-specific-purposes-esp.html, recuperado: marzo, 2017.

Freire, P, (2010). Cartas a quien pretende enseñar, México, siglo xxi editores s.a.

Gardner, H. (1995) Inteligencias múltiples, la teoría en la práctica, Barcelona, editorial Paidós, [en línea], disponible en: https://scholar.google.es/scholar?q=teor\%C3\%ADa+de+l

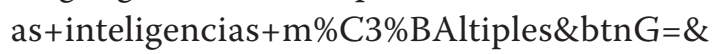
$\mathrm{hl}=$ es\&as_sdt $=0 \% 2 C 5 \&$ as_vis $=1$, recuperado: marzo, 2017

Hernández, R., Fernández C. \& Baptista P. (2006). "Metodología de la investigación", cuarta edición [en línea], disponible en: http://s3.amazonaws.com/academia.edu. documents/38758233/sampieri-et-al-metodologia-de-la-investigacion-4ta-edicion-sampieri-2006_ocr.pdf?AWSAccessKeyId=AKIAI WOWYYGZ2Y53UL3A\&Expires $=150128152$ $1 \&$ Signature $=$ KduWlAlp6Uuka710cQEMIH EES3U\%3D\&response-content-disposition =inline\%3B\%20filename\%3DSampieri-et-almetodologia-de-la-investi.pdf, recuperado: marzo, 2017.

Hernández, S. (2008). El modelo constructivista con las nuevas tecnologías, aplicado en el proceso de aprendizaje, en RUSC, Universities and Knowledge Society Journal, 5(2), 26-35.

Instituto Técnico Industrial Centro Don Bosco (CDB) (2006). Misión 2016, Bogotá, D.C. [en línea], disponible en: http://www.centrodonbosco.edu.co/inicio/quienes-somos/

Martínez, A. (2005). Creencias relativas al aprendizaje de una lengua extranjera, CAUCE, en Revista Internacional de Filología y su Didáctica, (28), 219-234.

Ministerio de Educación Nacional (MEN) (2003). Articulación de la educación con el 
mundo productivo o la formación de competencias laborales. Bogotá D.C., [en línea], disponible en: http: //www.mineducacion.gov. co/1621/articles-85777_archivo_pdf.pdf/articles-85777_archivo_pdf, recuperado: marzo, 2017.

Ministerio de Educación Nacional (MEN) (2006b). Ley 1064-2006, Diario Oficial No. 46341, [en línea], disponible en: http:// www.alcaldiabogota.gov.co/sisjur/normas/ Norma1.jsp?i=20854, recuperado: Marzo, 2017.Ministerio de Educación Nacional (MEN) (2006c). Decreto 3870/2006, [en línea], disponible en: http://cvc.cervantes.es/ ensenanza/biblioteca_ele/marco/cvc_mer. pdf, recuperado: marzo, 2017.

Ministerio de Educación Nacional (MEN) (2006a). Series guías No. 22, estándares básicos de competencias en lenguas extranjeras: inglés, formar en lenguas extranjeras: el reto, lo que necesitamos saber y saber hacer, [en línea], disponible en: www.colombiaaprende. edu.co/html/mediateca/1607/articles-115375_ archivo.pdf, recuperado: marzo, 2017.

Ministerio de Educación Nacional (2009). Decreto 4904/2009, [en línea], disponible en: http://www.mineducacion.gov.co/1621/articles-216551_archivo_pdf_decreto4904.pdf), recuperado: marzo, 2017.

Ministerio de Educación Nacional (2013). Orientaciones para la Implementación de Proyectos de Fortalecimiento de Inglés en las Entidades Territoriales. Bogotá D.C. Colombia, [en línea], disponible en: www. colombiaaprende.edu.co/html $/ \mathrm{microsi}$ tios/1752/articles-315518_recurso_5.pdf
Monterrey, I. T. (s.f.). Las Estrategias y Técnicas Didácticas en el Rediseño, [en línea], disponible en: http://sitios.itesm.mx/va/dide/ documentos/inf-doc/Est_y_tec.PDF, recuperado: marzo, 2017.

Moreno, J. (1994). Pensamiento, lenguaje y comunicación. Evaluación, teorías, modelos, actividades. Colección Papeles de Tertulia, Santafe de Bogotá.

Núñez, F.L. (2007). ¿Cómo se elabora un cuestionario? Barcelona, España. Material aportado en la especialización en Docencia Universitaria de la Universidad Militar Nueva Granada, 2017.

Patricio, T. \& María S. (2014). Competencia intercultural en la enseñanza de lenguas extranjeras, Porta Linguarum, [en línea], disponible en: http://digibug.ugr.es/bits-tream /10481/30491/1/Paricio_CompetenciaInterculturalLE.pdf., recuperado: Marzo, 2017.

Pérez, J. (2010, abril) "El seminario alemán una estrategia pedagógica para el estudiante", en Cultura, Educación y Sociedad, Volumen 1, Número 1, pp. 107-112.

Restrepo, B. (2005). “Aprendizaje basado en problemas (ABP): una innovación didáctica para la enseñanza universitaria", en Educación y Educadores, vol. 8, pp. 9-19.

Universidad de Los Andes. (s.f). "Metodología de la investigación", [en línea], disponible en: https://jofillop.files.wordpress.com/2011/03/ metodos-de-investigacion.pdf, recuperado: marzo, 2017.

Wagensberg J. (2007). El gozo intelectual, teoría y práctica sobre la inteligibilidad y la belleza, Tusquets editores S.A., España. 